NISSUNA UMANA INVESTIGAZIONE SI PUO DIMANDARE VERA SCIENZIA S'ESSA NON PASSA PER LE MATEMATICHE DIMOSTRAZIONI LEONARDO DA VINCI

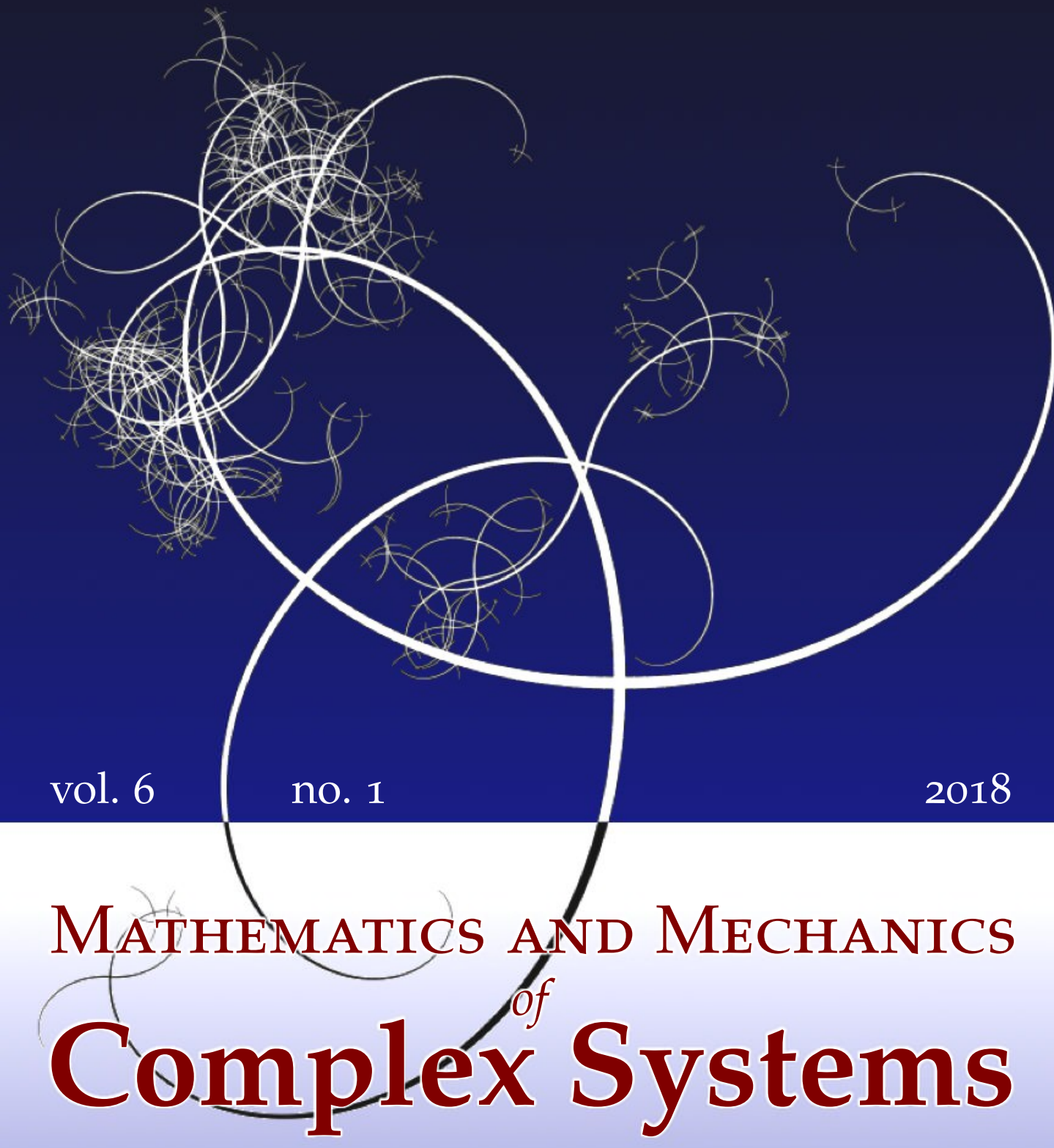

Micol Amar AND Roberto Gianni

ERROR ESTIMATE FOR A HOMOGENIZATION PROBLEM INVOLVING THE LAPLACE-BELTRAMI OPERATOR 


\title{
ERROR ESTIMATE FOR A HOMOGENIZATION PROBLEM INVOLVING THE LAPLACE-BELTRAMI OPERATOR
}

\author{
Micol AmAR AND Roberto GiAnNi
}

\begin{abstract}
In this paper we prove an error estimate for a model of heat conduction in composite materials having a microscopic structure arranged in a periodic array and thermally active membranes separating the heat-conductive phases.
\end{abstract}

\section{Introduction}

Heat and electrical conduction in composite materials has been widely investigated in recent years in the context of homogenization theory (see among others, e.g., [Amar et al. 2017a; 2017b; 2003; 2004; 2006; 2010; Auriault and Ene 1994; Bunoiu and Timofte 2016; Donato and Monsurrò 2001; Hummel 2000; Jose 2009; Timofte 2013]). In this paper we will focus on the study of models of heat conduction in composite materials used for encapsulation of electronic devices. This topic is attracting increasing interest among researchers, both from the point of view of applications and also in a more mathematical setting. In our previous paper [Amar and Gianni 2018b] (to which we refer for a more detailed physical description of the problem) a composite medium was taken into account, which was made of a hosting material with inclusions separated from their surroundings by a thermally active membrane.

Such a situation is consistent with many physical applications in which a material must be modified in a way such that its thermal conductivity is enhanced while preserving other material properties, e.g., ductility. This is, as stated above, the case of polymer encapsulation of electronic devices as well as, just to give an example, engine coolants. Specifically, in the first case, ductility of the material is required to fill the voids and the interstices among the electrical components by applying a moderate pressure. Polymers and rubbers have this property, but they do not display a satisfactory heat dissipation which, on the other hand, can be attained

\section{Communicated by Francesco dell'Isola.}

We would like to thank R. Lipton and P. Bisegna for some helpful discussions. Amar is a member of the Gruppo Nazionale per l'Analisi Matematica, la Probabilità e le loro Applicazioni (GNAMPA) of the Istituto Nazionale di Alta Matematica (INdAM).

MSC2010: 35B27, 35Q79.

Keywords: homogenization, asymptotic expansion, Laplace-Beltrami operator, heat conduction. 
by adding highly conductive nanoparticles. In some situations, these nanoparticles are enclosed in a membrane separating them from the surrounding medium. It is therefore only natural to investigate the influence of these membranes on the overall conductivity of the composite medium under different assumptions on the thermal behavior of these interfaces. The case of perfect or imperfect thermal contact, though interesting from the point of view of applications, is mathematically well known; for this reason we focused on the case in which the membrane is thermally active, e.g., a tangential heat diffusion takes place. In [Amar and Gianni 2018b] a macroscopic model was deduced, via the unfolding homogenization technique, assuming the periodicity of the microscopic structure, whose characteristic length is described by a small parameter $\varepsilon$. We make use of a sensible mathematical description of the behavior of the interfaces which are modeled by means of the Laplace-Beltrami operator (see, e.g., [Allaire et al. 1996; Andreucci et al. 2003]).

In this paper we complete the research started in [Amar and Gianni 2018b] providing an "error estimate" which enables us to evaluate the rate of convergence, with respect to $\varepsilon \rightarrow 0$, of the solution $u_{\varepsilon}$ of the microscopic (physical) problem to the solution $u_{0}$ of the macroscopic one. More precisely, we prove

$$
\begin{aligned}
\left\|u_{\varepsilon}-\left(u_{0}+\varepsilon u_{1}\right)\right\|_{L^{2}\left(0, T ; H^{1}(\Omega)\right)} & \leq \gamma \sqrt{\varepsilon}, \\
\left\|u_{\varepsilon}-u_{0}\right\|_{L^{2}\left(\Omega_{T}\right)} & \leq \gamma \sqrt{\varepsilon},
\end{aligned}
$$

for a proper constant $\gamma>0$ independent of $\varepsilon$, where $u_{1}$ is the so-called first corrector and is defined in (3-13).

To obtain this estimate we follow the classical approach given by the asymptotic expansions due to Bensoussan, Lions, and Papanicolaou [Bensoussan et al. 1978] which, under extra-regularity assumptions, gives an $H^{1}$-estimate for this error. The knowledge of the rate of convergence is a crucial tool for numerical applications. Moreover, we prove the symmetry and the strict positivity of the matrix describing the diffusivity of the macroscopic (homogenized) material. This last result is crucial to guarantee the well-posedness of the parabolic limit equation.

Though the results proved in this paper are along the same lines as other ones obtained in the framework of the homogenization theory, they are nevertheless of some mathematical interest due to the presence of the Laplace-Beltrami operator, which makes the computations a bit tricky.

The paper is organized as follows. In Section 2 we recall the definitions and some properties of the tangential operators (gradient, divergence, and LaplaceBeltrami operator), state our geometrical setting, and present our model. In Section 3, after having proved some energy inequalities, we follow the formal approach by Bensoussan, Lions, and Papanicolaou in order to introduce the cell functions and to guess the limit equation, proving the ellipticity of its principal part (see Theorem 3.1). 
Finally, in Section 4 taking advantage of the asymptotic expansions obtained in Section 3, we provide the error estimate (see Theorem 4.1).

\section{Preliminaries}

2.1. Tangential derivatives. Let $\phi$ be a $\mathcal{C}^{2}$-function, $\Phi$ a $\mathcal{C}^{2}$-vector function, and $S$ a smooth surface with normal unit vector $n$. We recall that the tangential gradient of $\phi$ is given by

$$
\nabla^{B} \phi=\nabla \phi-(n \cdot \nabla \phi) n
$$

and the tangential divergence of $\boldsymbol{\Phi}$ is given by

$$
\begin{aligned}
\operatorname{div}^{B} \boldsymbol{\Phi}=\operatorname{div} \boldsymbol{\Phi}-\left(n \cdot \nabla \boldsymbol{\Phi}_{i}\right) n_{i} & -(\operatorname{div} n)(n \cdot \boldsymbol{\Phi}) \\
& =\operatorname{div}^{B}(\boldsymbol{\Phi}-(n \cdot \boldsymbol{\Phi}) n)=\operatorname{div}(\boldsymbol{\Phi}-(n \cdot \boldsymbol{\Phi}) n),
\end{aligned}
$$

where, taking into account the smoothness of $S$, the normal vector $n$ can be naturally defined in a small neighborhood of $S$ as $\nabla d /|\nabla d|$, where $d$ is the signed distance from $S$. Moreover, we define the Laplace-Beltrami operator as

$$
\Delta^{B} \phi=\operatorname{div}^{B}\left(\nabla^{B} \phi\right),
$$

so that by (2-1) and (2-2) we get that the Laplace-Beltrami operator can be written as

$$
\begin{aligned}
\Delta^{B} \phi & =\Delta \phi-n^{t} \nabla^{2} \phi n-(n \cdot \nabla \phi) \operatorname{div} n \\
& =\left(\delta_{i j}-n_{i} n_{j}\right) \partial_{i j}^{2} \phi-n_{j} \partial_{j} \phi \partial_{i} n_{i}=(I-n \otimes n)_{i j} \partial_{i j}^{2} \phi-(n \cdot \nabla \phi) \operatorname{div} n,
\end{aligned}
$$

where $\nabla^{2} \phi$ stands for the Hessian matrix of $\phi$. Finally, we recall that on a regular surface $S$ with no boundary (i.e., when $\partial S=\varnothing$ ) we have

$$
\int_{S} \operatorname{div}^{B} \boldsymbol{\Phi} \mathrm{d} \sigma=0 .
$$

2.2. Geometrical setting. The typical periodic geometrical setting is displayed in Figure 1. Here we give, for the sake of clarity, its detailed formal definition.

Let us introduce a periodic open subset $E$ of $\mathbb{R}^{N}$, so that $E+z=E$ for all $z \in \mathbb{Z}^{N}$. We employ the notation $Y=(0,1)^{N}$, and $E_{\text {int }}=E \cap Y, E_{\text {out }}=Y \backslash \bar{E}$, and $\Gamma=\partial E \cap \bar{Y}$. We assume that $E_{\text {out }}$ is connected and $\Gamma \cap \partial Y=\varnothing$.

Let $\Omega$ be an open connected bounded subset of $\mathbb{R}^{N}$; for all $\varepsilon>0$ define $\Omega_{\text {int }}^{\varepsilon}=$ $\Omega \cap \varepsilon E$ and $\Omega_{\text {out }}^{\varepsilon}=\Omega \backslash \overline{\varepsilon E}$, so that $\Omega=\Omega_{\text {int }}^{\varepsilon} \cup \Omega_{\text {out }}^{\varepsilon} \cup \Gamma^{\varepsilon}$, where $\Omega_{\text {int }}^{\varepsilon}$ and $\Omega_{\text {out }}^{\varepsilon}$ are two disjoint open subsets of $\Omega$, and $\Gamma^{\varepsilon}=\partial \Omega_{\text {int }}^{\varepsilon} \cap \Omega=\partial \Omega_{\text {out }}^{\varepsilon} \cap \Omega$. The regions $\Omega_{\text {out }}^{\varepsilon}$ and $\Omega_{\text {int }}^{\varepsilon}$ correspond to the outer phase and the inclusions, respectively, while $\Gamma^{\varepsilon}$ is the interface. We also assume that $\Omega$ and $E$ have regular boundary, and we stipulate that $\operatorname{dist}\left(\Gamma^{\varepsilon}, \partial \Omega\right) \geq \gamma_{0} \varepsilon$, for a suitable $\gamma_{0}>0$. To this purpose, for each $\varepsilon$, we are ready to remove the inclusions in all the cells which are not completely contained in $\Omega$ (see Figure 1). This assumption is in accordance with our previous 

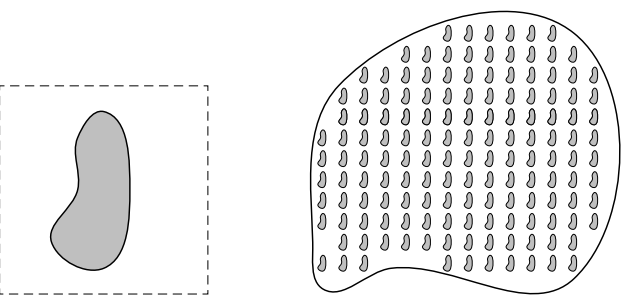

Figure 1. Left: the periodic cell $Y . E_{\text {int }}$ is the shaded region, and $E_{\text {out }}$ is the white region. Right: the region $\Omega$.

papers [Amar et al. 2004; 2006; 2009a; 2009b; 2010; 2013], and maybe it can be dropped as in [Allaire and Murat 1993; Cioranescu et al. 2012]; nevertheless, we will not pursue this line of investigation in this paper.

Moreover, let $v$ denote the normal unit vector to $\Gamma$ pointing into $E_{\text {out }}$, extended by periodicity to the whole of $\mathbb{R}^{N}$, so that $v_{\varepsilon}(x)=v(x / \varepsilon)$ denotes the normal unit vector to $\Gamma^{\varepsilon}$ pointing into $\Omega_{\text {out }}^{\varepsilon}$.

Finally, given $T>0$, we denote $\Omega_{T}=\Omega \times(0, T)$. More generally, for any spatial domain $G$, we denote $G_{T}=G \times(0, T)$.

2.3. Position of the problem. Let $\mu^{\varepsilon}, \lambda^{\varepsilon}: \Omega \rightarrow \mathbb{R}$ be defined as

$$
\begin{array}{llll}
\lambda^{\varepsilon}=\lambda_{\text {int }} & \text { in } \Omega_{\text {int }}^{\varepsilon}, & \lambda^{\varepsilon}=\lambda_{\text {out }} & \text { in } \Omega_{\text {out }}^{\varepsilon}, \\
\mu^{\varepsilon}=\mu_{\text {int }} & \text { in } \Omega_{\text {int }}^{\varepsilon}, & \mu^{\varepsilon}=\mu_{\text {out }} & \text { in } \Omega_{\text {out }}^{\varepsilon} .
\end{array}
$$

For every $\varepsilon>0$, we consider the problem for $u_{\varepsilon}(x, t)$ given by

$$
\begin{aligned}
\mu^{\varepsilon} \frac{\partial u_{\varepsilon}}{\partial t}-\operatorname{div}\left(\lambda^{\varepsilon} \nabla u_{\varepsilon}\right) & =0 & & \text { in }\left(\Omega_{\text {int }}^{\varepsilon} \cup \Omega_{\text {out }}^{\varepsilon}\right) \times(0, T), \\
{\left[u_{\varepsilon}\right] } & =0 & & \text { on } \Gamma_{T}^{\varepsilon}, \\
\varepsilon \alpha \frac{\partial u_{\varepsilon}}{\partial t}-\varepsilon \beta \Delta^{B} u_{\varepsilon} & =\left[\lambda^{\varepsilon} \nabla u_{\varepsilon} \cdot v_{\varepsilon}\right] & & \text { on } \Gamma_{T}^{\varepsilon}, \\
u_{\varepsilon}(x, t) & =0 & & \text { on } \partial \Omega \times(0, T), \\
u_{\varepsilon}(x, 0) & =\bar{u}_{0}(x) & & \text { in } \Omega,
\end{aligned}
$$

where we denote

$$
\left[u_{\varepsilon}\right]=u_{\varepsilon}^{\text {out }}-u_{\varepsilon}^{\text {int }},
$$

and the same notation is employed also for other quantities. We assume that all the constants $\mu_{\text {int }}, \mu_{\text {out }}, \lambda_{\text {int }}, \lambda_{\text {out }}, \alpha, \beta$ involved in (2-6) and (2-8) are strictly positive.

Since problem (2-6)-(2-10) is not standard, in order to define a proper notion of weak solution, we will need to introduce some suitable function spaces. To this purpose and for later use, we will denote by $H_{B}^{1}\left(\Gamma^{\varepsilon}\right)$ the space of Lebesguemeasurable functions $u: \Gamma^{\varepsilon} \rightarrow \mathbb{R}$ such that $u \in L^{2}\left(\Gamma^{\varepsilon}\right)$ and $\nabla^{B} u \in L^{2}\left(\Gamma^{\varepsilon}\right)$. Let 
us also set

$$
\mathcal{X}_{0}^{\varepsilon}(\Omega):=H_{0}^{1}(\Omega) \cap H_{B}^{1}\left(\Gamma^{\varepsilon}\right)
$$

Definition 2.1. We say that $u_{\varepsilon} \in L^{2}\left(0, T ; \mathcal{X}_{0}^{\varepsilon}(\Omega)\right)$ is a weak solution of problem $(2-6)-(2-10)$ if

$$
\begin{aligned}
& -\int_{0}^{T} \int_{\Omega} \mu^{\varepsilon} u_{\varepsilon} \frac{\partial \phi}{\partial \tau} \mathrm{d} x \mathrm{~d} \tau+\int_{0}^{T} \int_{\Omega} \lambda^{\varepsilon} \nabla u_{\varepsilon} \cdot \nabla \phi \mathrm{d} x \mathrm{~d} \tau \\
& -\varepsilon \alpha \int_{0}^{T} \int_{\Gamma^{\varepsilon}} u_{\varepsilon} \frac{\partial \phi}{\partial \tau} \mathrm{d} \sigma \mathrm{d} \tau+\varepsilon \beta \int_{0}^{T} \int_{\Gamma^{\varepsilon}} \nabla^{B} u_{\varepsilon} \cdot \nabla^{B} \phi \mathrm{d} \sigma \mathrm{d} \tau \\
& \quad=\int_{\Omega} \mu^{\varepsilon} \bar{u}_{0} \phi(x, 0) \mathrm{d} x+\varepsilon \alpha \int_{\Gamma^{\varepsilon}} \bar{u}_{0} \phi(x, 0) \mathrm{d} \sigma,
\end{aligned}
$$

for every test function $\phi \in \mathcal{C}^{\infty}\left(\Omega_{T}\right)$ such that $\phi$ has compact support in $\Omega$ for every $t \in(0, T)$ and $\phi(\cdot, T)=0$ in $\Omega$.

If $u_{\varepsilon}$ is smooth, by (2-4) it follows that (2-8) can be written in the form

$$
\varepsilon \alpha \frac{\partial u_{\varepsilon}}{\partial t}-\varepsilon \beta\left(\Delta u_{\varepsilon}-v_{\varepsilon}^{t} \nabla^{2} u_{\varepsilon} v_{\varepsilon}-\left(v_{\varepsilon} \cdot \nabla u_{\varepsilon}\right) \operatorname{div} v_{\varepsilon}\right)=\left[\lambda \nabla u_{\varepsilon} \cdot v_{\varepsilon}\right] \quad \text { on } \Gamma^{\varepsilon},
$$

where, as in (2-4), $\nabla^{2} u_{\varepsilon}$ stands for the Hessian matrix of $u_{\varepsilon}$. By [Amar and Gianni 2018a, Theorem 4.2], for every $\varepsilon>0$, problem (2-6)-(2-10) admits a unique solution $u_{\varepsilon} \in L^{2}\left(0, T ; \mathcal{X}_{0}^{\varepsilon}(\Omega)\right) \cap \mathcal{C}^{0}\left([0, T] ; L^{2}(\Omega) \cap L^{2}\left(\Gamma^{\varepsilon}\right)\right)$, if $\bar{u}_{0} \in H_{0}^{1}(\Omega)$.

Finally, it will be useful in the sequel to also define $\mu, \lambda: Y \rightarrow \mathbb{R}$ as

$$
\begin{aligned}
& \lambda=\lambda_{\text {int }} \quad \text { in } E_{\text {int }}, \quad \lambda=\lambda_{\text {out }} \quad \text { in } E_{\text {out }}, \\
& \mu=\mu_{\text {int }} \quad \text { in } E_{\text {int }}, \quad \mu=\mu_{\text {out }} \quad \text { in } E_{\text {out }} \text {. }
\end{aligned}
$$

\section{Homogenization of the microscopic problem}

In the following, we will assume that the initial data satisfies

$$
\bar{u}_{0} \in H_{0}^{1}(\Omega) \cap H^{2}(\Omega) .
$$

By the trace inequality [Amar and Gianni 2018b, Proposition 1; Amar et al. 2004, proof of Lemma 7.1] we get that $\bar{u}_{0}$ satisfies

$$
\varepsilon \int_{\Gamma^{\varepsilon}}\left|\bar{u}_{0}\right|^{2} \mathrm{~d} \sigma \leq \gamma, \quad \varepsilon \int_{\Gamma^{\varepsilon}}\left|\nabla^{B} \bar{u}_{0}\right|^{2} \mathrm{~d} \sigma \leq \gamma,
$$

where $\gamma>0$ is independent of $\varepsilon$. Notice that, for our purposes, it should be enough to assume that $\bar{u}_{0} \in H_{0}^{1}(\Omega)$ and satisfies (3-2), but we prefer to assume (3-1) since it is reasonable to choose $\bar{u}_{0}$ not depending on $\varepsilon$.

We are interested in understanding the limiting behavior of the heat potential $u_{\varepsilon}$ when $\varepsilon \rightarrow 0$; this leads us to look at the homogenization limit of problem (2-6)(2-10). 
To this purpose, we first obtain some energy estimates for the heat potential $u_{\varepsilon}$. Multiplying (2-6) by $u_{\varepsilon}$ and integrating, formally, by parts, we obtain

$$
\begin{aligned}
\frac{1}{2} \int_{0}^{t} \int_{\Omega} \mu^{\varepsilon} \frac{\partial u_{\varepsilon}^{2}}{\partial \tau} \mathrm{d} x \mathrm{~d} \tau+\int_{0}^{t} \int_{\Omega} \lambda^{\varepsilon}\left|\nabla u_{\varepsilon}\right|^{2} \mathrm{~d} x \mathrm{~d} \tau \\
+\frac{\varepsilon \alpha}{2} \int_{0}^{t} \int_{\Gamma^{\varepsilon}} \frac{\partial u_{\varepsilon}^{2}}{\partial \tau} \mathrm{d} \sigma \mathrm{d} \tau+\varepsilon \beta \int_{0}^{t} \int_{\Gamma^{\varepsilon}}\left|\nabla^{B} u_{\varepsilon}\right|^{2}(x) \mathrm{d} \sigma \mathrm{d} \tau=0 .
\end{aligned}
$$

Then, evaluating the time integral and taking into account the initial condition (2-10), we obtain, for all $0<t<T$,

$$
\begin{aligned}
\frac{1}{2} \int_{\Omega} \mu^{\varepsilon} u_{\varepsilon}^{2}(t) \mathrm{d} x+\int_{0}^{t} \int_{\Omega} \lambda^{\varepsilon}\left|\nabla u_{\varepsilon}\right|^{2} \mathrm{~d} x \mathrm{~d} \tau & +\frac{\varepsilon \alpha}{2} \int_{\Gamma^{\varepsilon}} u_{\varepsilon}^{2}(t) \mathrm{d} \sigma+\varepsilon \beta \int_{0}^{t} \int_{\Gamma^{\varepsilon}}\left|\nabla^{B} u_{\varepsilon}\right|^{2} \mathrm{~d} \sigma \mathrm{d} \tau \\
& =\frac{1}{2} \int_{\Omega} \mu^{\varepsilon} \bar{u}_{0}^{2} \mathrm{~d} x+\frac{\varepsilon \alpha}{2} \int_{\Gamma^{\varepsilon}} \bar{u}_{0}^{2} \mathrm{~d} \sigma .
\end{aligned}
$$

By (3-2) the right-hand side of (3-4) is stable as $\varepsilon \rightarrow 0$; hence,

$$
\begin{aligned}
\sup _{t \in(0, T)} \int_{\Omega} u_{\varepsilon}^{2}(t) \mathrm{d} x & +\int_{0}^{T} \int_{\Omega}\left|\nabla u_{\varepsilon}\right|^{2} \mathrm{~d} x \mathrm{~d} \tau \\
& +\sup _{t \in(0, T)} \varepsilon \int_{\Gamma^{\varepsilon}} u_{\varepsilon}^{2}(t) \mathrm{d} \sigma+\varepsilon \int_{0}^{T} \int_{\Gamma^{\varepsilon}}\left|\nabla^{B} u_{\varepsilon}\right|^{2} \mathrm{~d} \sigma \mathrm{d} \tau \leq \gamma,
\end{aligned}
$$

where $\gamma$ is a constant independent of $\varepsilon$.

Notice that inequality (3-5) implies that there exists a function $u$ belonging to $L^{2}\left(0, T ; H_{0}^{1}(\Omega)\right)$ such that, up to a subsequence, $u_{\varepsilon} \rightarrow u$ weakly in $L^{2}\left(0, T ; H_{0}^{1}(\Omega)\right)$. It will be our purpose to characterize the limit function $u$.

3.1. The two-scale expansion. We summarize here, to establish the notation, some well known asymptotic expansions needed in the two-scale method (see, e.g., [Bensoussan et al. 1978; Sánchez-Palencia 1980]), when applied to stationary or evolutive problems involving second-order partial differential equations. Introduce the microscopic variables $y \in Y$ and $y=x / \varepsilon$, and assume

$$
u_{\varepsilon}=u_{\varepsilon}(x, y, t)=u_{0}(x, y, t)+\varepsilon u_{1}(x, y, t)+\varepsilon^{2} u_{2}(x, y, t)+\cdots .
$$

Note that $u_{0}, u_{1}, u_{2}$ are periodic in $y$, and $u_{1}, u_{2}$ are assumed to have zero integral average over $Y$. Recalling that

$$
\operatorname{div}=\frac{1}{\varepsilon} \operatorname{div}_{y}+\operatorname{div}_{x}, \quad \nabla=\frac{1}{\varepsilon} \nabla_{y}+\nabla_{x},
$$

we compute

$$
\nabla u_{\varepsilon}=\frac{1}{\varepsilon} \nabla_{y} u_{0}+\left(\nabla_{x} u_{0}+\nabla_{y} u_{1}\right)+\varepsilon\left(\nabla_{y} u_{2}+\nabla_{x} u_{1}\right)+\cdots,
$$


and

$$
\Delta u_{\varepsilon}=\frac{1}{\varepsilon^{2}} A_{0} u_{0}+\frac{1}{\varepsilon}\left(A_{0} u_{1}+A_{1} u_{0}\right)+\left(A_{0} u_{2}+A_{1} u_{1}+A_{2} u_{0}\right)+\cdots,
$$

where

$$
A_{0}=\Delta_{y}, \quad A_{1}=\operatorname{div}_{y} \nabla_{x}+\operatorname{div}_{x} \nabla_{y}, \quad A_{2}=\Delta_{x}
$$

Moreover, recalling (2-3) and taking into account that the normal vector $v_{\varepsilon}$ depends only on the microscopic variable, we also obtain

$$
\Delta^{B} u_{\varepsilon}=\frac{1}{\varepsilon^{2}} A_{0}^{B} u_{0}+\frac{1}{\varepsilon}\left(A_{0}^{B} u_{1}+A_{1}^{B} u_{0}\right)+\left(A_{0}^{B} u_{2}+A_{1}^{B} u_{1}+A_{2}^{B} u_{0}\right)+\cdots,
$$

where

$$
\begin{aligned}
& A_{0}^{B}=\Delta_{y}^{B}, \quad A_{2}^{B}=\Delta_{x}^{B} \\
& A_{1}^{B}=\operatorname{div}_{x}^{B} \nabla_{y}^{B}+\operatorname{div}_{y}^{B} \nabla_{x}^{B}=2(I-v \otimes v)_{i j} \partial_{x_{i} y_{j}}^{2}-\left(\operatorname{div}_{y} v\right) v \cdot \nabla_{x} .
\end{aligned}
$$

Substituting in (2-6)-(2-10) the expansion (3-6), and using (3-7)-(3-12), one readily obtains, by matching corresponding powers of $\varepsilon$, that $u_{0}$ solves $\left[u_{0}\right]=0$ on $\Gamma$, and

$$
\mathcal{P}_{0}\left[u_{0}\right]: \begin{cases}-\lambda \Delta_{y} u_{0}=0 & \text { in } E_{\text {int }}, E_{\text {out }}, \\ \beta \Delta_{y}^{B} u_{0}+\left[\lambda \nabla_{y} u_{0} \cdot v\right]=0 & \text { on } \Gamma\end{cases}
$$

By the equality

$$
\begin{aligned}
0 & =\int_{Y} \lambda\left|\nabla_{y} u_{0}\right|^{2} \mathrm{~d} y+\int_{\Gamma}\left[\lambda \nabla_{y} u_{0} \cdot v\right] u_{0} \mathrm{~d} \sigma \\
& =\int_{Y} \lambda\left|\nabla_{y} u_{0}\right|^{2} \mathrm{~d} y-\int_{\Gamma} \beta \Delta_{y}^{B} u_{0} u_{0} \mathrm{~d} \sigma \\
& =\int_{Y} \lambda\left|\nabla_{y} u_{0}\right|^{2} \mathrm{~d} y+\int_{\Gamma} \beta\left|\nabla_{y}^{B} u_{0}\right|^{2} \mathrm{~d} \sigma,
\end{aligned}
$$

we obtain that $u_{0}$ is independent of $y$, i.e., $u_{0}=u_{0}(x, t)$.

Moreover, $u_{1}$ satisfies $\left[u_{1}\right]=0$ on $\Gamma$, and

$$
\mathcal{P}_{1}\left[u_{1}\right]: \begin{cases}-\lambda \Delta_{y} u_{1}=0 & \text { in } E_{\text {int }}, E_{\text {out }}, \\ \beta \Delta_{y}^{B} u_{1}+\left[\lambda \nabla_{y} u_{1} \cdot v\right]=-\beta\left(\operatorname{div}_{y}^{B} \nabla_{x}^{B} u_{0}\right)-\left[\lambda \nabla_{x} u_{0} \cdot v\right] & \text { on } \Gamma .\end{cases}
$$

Following a classical approach, we introduce the factorization

$$
u_{1}(x, y, t)=-\chi(y) \cdot \nabla_{x} u_{0}(x, t)=-\chi_{h}(y) \frac{\partial u_{0}}{\partial x_{h}}(x, t), \quad h=1, \ldots, N,
$$


for a vector function $\chi: Y \rightarrow \mathbb{R}^{N}$, whose components $\chi_{h}$ satisfy

$$
\begin{aligned}
-\lambda \operatorname{div}_{y}\left(\nabla_{y} \chi_{h}-\boldsymbol{e}_{h}\right) & =0 & & \text { in } E_{\mathrm{int}}, E_{\mathrm{out}}, \\
\beta \Delta_{y}^{B}\left(\chi_{h}-y_{h}\right) & =-\left[\lambda\left(\nabla_{y} \chi_{h}-\boldsymbol{e}_{h}\right) \cdot \nu\right] & & \text { on } \Gamma, \\
{\left[\chi_{h}\right] } & =0 & & \text { on } \Gamma .
\end{aligned}
$$

The functions $\chi_{h}$ are also required to be periodic in $Y$, with zero integral average on $Y$ (here, $\boldsymbol{e}_{h}$ denotes the $h$ vector of the canonical basis of $\mathbb{R}^{N}$ ). We note that (Amar and Gianni 2018a, Theorem 5.1 and Remark 5.3] assures existence and uniqueness of the cell functions $\chi_{h} \in \mathcal{C}_{\#}^{\infty}(Y)$, for $h=1, \ldots, N$ (here and in the following, the subscript \# denotes the $Y$-periodicity).

Finally, $u_{2}$ solves $\left[u_{2}\right]=0$ on $\Gamma$, and

$$
\mathcal{P}_{2}\left[u_{2}\right]:\left\{\begin{array}{cc}
-\lambda \Delta_{y} u_{2}=-\mu u_{0 t}+\lambda \Delta_{x} u_{0}+2 \lambda \frac{\partial^{2} u_{1}}{\partial x_{j} \partial y_{j}} & \text { in } E_{\mathrm{int}}, E_{\mathrm{out}}, \\
\beta \Delta_{y}^{B} u_{2}+\left[\lambda \nabla_{y} u_{2} \cdot v\right]=\alpha u_{0 t}-\beta \Delta_{x}^{B} u_{0}-\beta \operatorname{div}_{x}^{B} \nabla_{y}^{B} u_{1} & \\
-\beta \operatorname{div}_{y}^{B} \nabla_{x}^{B} u_{1}-\left[\lambda \nabla_{x} u_{1} \cdot v\right] & \text { on } \Gamma .
\end{array}\right.
$$

The limiting equation for $u_{0}$ is finally obtained as a compatibility condition for $\mathcal{P}_{2}\left[u_{2}\right]$, and amounts to

$$
\begin{aligned}
& \int_{Y}\left(-\mu u_{0 t}+\lambda \Delta_{x} u_{0}+2 \lambda \frac{\partial^{2} u_{1}}{\partial x_{j} \partial y_{j}}\right) \mathrm{d} y=\int_{\Gamma}\left[\lambda \nabla_{y} u_{2} \cdot v\right] \mathrm{d} \sigma \\
& =\int_{\Gamma}\left(\alpha u_{0 t}-\left[\lambda \nabla_{x} u_{1} \cdot v\right]-\beta \Delta_{y}^{B} u_{2}-\beta \Delta_{x}^{B} u_{0}-\beta \operatorname{div}_{x}^{B} \nabla_{y}^{B} u_{1}-\beta \operatorname{div}_{y}^{B} \nabla_{x}^{B} u_{1}\right) \mathrm{d} \sigma .
\end{aligned}
$$

We now replace the factorization (3-13) in the previous equality, and we take into account that

$$
\begin{aligned}
2 \int_{Y} \lambda \frac{\partial^{2} u_{1}}{\partial x_{j} \partial y_{j}} \mathrm{~d} y & =-2 \int_{\Gamma}\left[\lambda \nabla_{x} u_{1} \cdot v\right] \mathrm{d} \sigma \\
-\int_{\Gamma}\left[\lambda \nabla_{x} u_{1} \cdot v\right] \mathrm{d} \sigma & =\operatorname{div}\left(\left(\int_{\Gamma}[\lambda](v \otimes \chi) \mathrm{d} \sigma\right) \nabla u_{0}\right), \\
-\int_{\Gamma} \beta \Delta_{y}^{B} u_{2} \mathrm{~d} \sigma & =0, \\
-\int_{\Gamma} \beta \Delta_{x}^{B} u_{0} \mathrm{~d} \sigma & =-\beta|\Gamma| \Delta u_{0}+\operatorname{div}\left(\left(\int_{\Gamma} \beta(v \otimes v) \mathrm{d} \sigma\right) \nabla u_{0}\right), \\
-\int_{\Gamma} \beta \operatorname{div}_{x}^{B} \nabla_{y}^{B} u_{1} \mathrm{~d} \sigma & =\operatorname{div}\left(\left(\int_{\Gamma} \beta(I-v \otimes v) \nabla_{y} \chi \mathrm{d} \sigma\right) \nabla u_{0}\right), \\
-\int_{\Gamma} \beta \operatorname{div}_{y}^{B} \nabla_{x}^{B} u_{1} \mathrm{~d} \sigma & =0,
\end{aligned}
$$


where (3-23) follows from (2-5), since $\Gamma$ has no boundary. Hence, we obtain for the homogenized solution $u_{0}$ the parabolic equation

$$
\tilde{\mu} u_{0 t}-\operatorname{div}\left(\left(\lambda_{0} I+A^{\text {hom }}\right) \nabla u_{0}\right)=0 \quad \text { in } \Omega_{T},
$$

where

$$
\begin{aligned}
\tilde{\mu} & =\mu_{\text {int }}\left|E_{\text {int }}\right|+\mu_{\text {out }}\left|E_{\text {out }}\right|+\alpha|\Gamma|, \\
\lambda_{0} & =\lambda_{\text {int }}\left|E_{\text {int }}\right|+\lambda_{\text {out }}\left|E_{\text {out }}\right| \\
A^{\text {hom }} & =\int_{\Gamma}[\lambda](\nu \otimes \chi) \mathrm{d} \sigma+\beta \int_{\Gamma}\left((I-v \otimes v)+(\nu \otimes v) \nabla_{y} \chi-\nabla_{y} \chi\right) \mathrm{d} \sigma \\
& =\int_{\Gamma}[\lambda](\nu \otimes \chi) \mathrm{d} \sigma-\beta \int_{\Gamma} \nabla_{y}^{B}(\chi-y) \mathrm{d} \sigma .
\end{aligned}
$$

Clearly, (3-24) must be complemented with a boundary and an initial condition which are $u_{0}=0$ on $\partial \Omega \times(0, T)$ and $u_{0}(x, 0)=\bar{u}_{0}(x)$ in $\Omega$, respectively, as follows from the microscopic problem (2-6)-(2-10). Indeed, by (3-5) we obtain that $\left\{u_{\varepsilon}\right\}$ converges weakly in $L^{2}\left(0, T ; H_{0}^{1}(\Omega)\right)$, which implies the weak convergence of the trace on $\partial \Omega$, while the initial data is already included in the weak formulation of the problem.

Theorem 3.1. The matrix $\lambda_{0} I+A^{\mathrm{hom}}$ is symmetric and positive-definite.

Proof. We first prove the symmetry. By (2-1), we have

$$
-\int_{\Gamma} \nabla_{y}^{B} y_{h} \cdot \nabla_{y}^{B} \chi_{j} \mathrm{~d} \sigma=-\int_{\Gamma}\left(\boldsymbol{e}_{h}-v_{h} \nu\right) \cdot \nabla_{y}^{B} \chi_{j} \mathrm{~d} \sigma=-\int_{\Gamma}\left(\nabla_{y}^{B} \chi_{j}\right)_{h} \mathrm{~d} \sigma
$$

then, taking into account (3-14)-(3-16), we obtain

$$
\begin{aligned}
0 & =-\int_{Y} \lambda \Delta_{y}\left(\chi_{h}-y_{h}\right) \chi_{j} \mathrm{~d} y \\
= & \int_{Y} \lambda \nabla_{y}\left(\chi_{h}-y_{h}\right) \cdot \nabla_{y} \chi_{j} \mathrm{~d} y-\beta \int_{\Gamma} \Delta_{y}^{B}\left(\chi_{h}-y_{h}\right) \chi_{j} \mathrm{~d} \sigma \\
= & \int_{Y} \lambda \nabla_{y} \chi_{h} \cdot \nabla_{y} \chi_{j} \mathrm{~d} y-\int_{Y} \lambda \boldsymbol{e}_{h} \cdot \nabla_{y} \chi_{j} \mathrm{~d} y+\beta \int_{\Gamma} \nabla_{y}^{B}\left(\chi_{h}-y_{h}\right) \cdot \nabla_{y}^{B} \chi_{j} \mathrm{~d} \sigma \\
= & \int_{Y} \lambda \nabla_{y} \chi_{h} \cdot \nabla_{y} \chi_{j} \mathrm{~d} y+\int_{\Gamma}[\lambda] v_{h} \chi_{j} \mathrm{~d} \sigma \\
& \quad+\beta \int_{\Gamma} \nabla_{y}^{B} \chi_{h} \nabla_{y}^{B} \chi_{j} \mathrm{~d} \sigma-\beta \int_{\Gamma} \nabla_{y}^{B} y_{h} \nabla_{y}^{B} \chi_{j} \mathrm{~d} \sigma \\
= & \int_{Y} \lambda \nabla_{y} \chi_{h} \cdot \nabla_{y} \chi_{j} \mathrm{~d} y+\int_{\Gamma}[\lambda] v_{h} \chi_{j} \mathrm{~d} \sigma \\
& +\beta \int_{\Gamma} \nabla_{y}^{B} \chi_{h} \nabla_{y}^{B} \chi_{j} \mathrm{~d} \sigma-\beta \int_{\Gamma}\left(\nabla_{y}^{B} \chi_{j}\right)_{h} \mathrm{~d} \sigma .
\end{aligned}
$$


From (3-25) and (3-27), we can rewrite

$$
A^{\mathrm{hom}}=\int_{\Gamma} \beta(I-\nu \otimes \nu) \mathrm{d} \sigma-\int_{Y} \lambda\left(\nabla_{y} \chi \otimes \nabla_{y} \chi\right) \mathrm{d} y-\int_{\Gamma} \beta\left(\nabla_{y}^{B} \chi \otimes \nabla_{y}^{B} \chi\right) \mathrm{d} \sigma,
$$

which gives the symmetry of the matrix $A^{\text {hom }}$ and hence the symmetry of the whole matrix $\lambda_{0} I+A^{\text {hom }}$.

Let us now prove that it is also positive-definite. Firstly, we observe that, using (3-26) and (3-27), we obtain

$$
\begin{aligned}
& \int_{Y} \lambda \nabla\left(\chi_{h}-y_{h}\right) \cdot \nabla\left(\chi_{j}-y_{j}\right) \mathrm{d} y+\beta \int_{\Gamma} \nabla_{y}^{B}\left(\chi_{h}-y_{h}\right) \nabla_{y}^{B}\left(\chi_{j}-y_{j}\right) \mathrm{d} \sigma \\
& =\int_{Y} \lambda \nabla \chi_{h} \cdot \nabla \chi_{j} \mathrm{~d} y+\int_{Y} \lambda \boldsymbol{e}_{h} \cdot \boldsymbol{e}_{j} \mathrm{~d} y-\int_{Y} \lambda \nabla \chi_{h} \cdot \boldsymbol{e}_{j} \mathrm{~d} y-\int_{Y} \lambda \nabla \chi_{j} \cdot \boldsymbol{e}_{h} \mathrm{~d} y \\
& +\beta \int_{\Gamma} \nabla^{B} \chi_{h} \cdot \nabla^{B} \chi_{j} \mathrm{~d} \sigma+\beta \int_{\Gamma} \nabla^{B} y_{h} \cdot \nabla^{B} y_{j} \mathrm{~d} \sigma \\
& -\beta \int_{\Gamma} \nabla^{B} \chi_{h} \cdot \nabla^{B} y_{j} \mathrm{~d} \sigma-\beta \int_{\Gamma} \nabla^{B} \chi_{j} \cdot \nabla^{B} y_{h} \mathrm{~d} \sigma \\
& =\int_{Y} \lambda \nabla \chi_{h} \cdot \nabla \chi_{j} \mathrm{~d} y+\int_{Y} \lambda \delta_{h j} \mathrm{~d} y+\int_{\Gamma}[\lambda] \chi_{h} v_{j} \mathrm{~d} \sigma+\int_{\Gamma}[\lambda] \chi_{j} v_{h} \mathrm{~d} \sigma \\
& +\beta \int_{\Gamma} \nabla^{B} \chi_{h} \cdot \nabla^{B} \chi_{j} \mathrm{~d} \sigma+\beta \int_{\Gamma} \nabla^{B} y_{h} \cdot \nabla^{B} y_{j} \mathrm{~d} \sigma \\
& -\beta \int_{\Gamma}\left(\nabla_{y}^{B} \chi_{h}\right)_{j} \mathrm{~d} \sigma-\beta \int_{\Gamma}\left(\nabla_{y}^{B} \chi_{j}\right)_{h} \mathrm{~d} \sigma \\
& =\int_{Y} \lambda \nabla \chi_{h} \cdot \nabla \chi_{j} \mathrm{~d} y+\int_{Y} \lambda \delta_{h j} \mathrm{~d} y+\beta \int_{\Gamma} \nabla^{B} \chi_{h} \cdot \nabla^{B} \chi_{j} \mathrm{~d} \sigma+\beta \int_{\Gamma} \nabla^{B} y_{h} \cdot \nabla^{B} y_{j} \mathrm{~d} \sigma \\
& -2 \int_{Y} \lambda \nabla \chi_{h} \cdot \nabla \chi_{j} \mathrm{~d} y-2 \beta \int_{\Gamma} \nabla^{B} \chi_{h} \nabla^{B} \chi_{j} \mathrm{~d} \sigma \\
& =\int_{Y} \lambda \delta_{h j} \mathrm{~d} y-\int_{Y} \lambda \nabla \chi_{h} \cdot \nabla \chi_{j} \mathrm{~d} y+\beta \int_{\Gamma}\left(\delta_{h j}-v_{h} v_{j}\right) \mathrm{d} \sigma-\beta \int_{\Gamma} \nabla^{B} \chi_{h} \nabla^{B} \chi_{j} \mathrm{~d} \sigma .
\end{aligned}
$$

Then, we can rewrite

$$
\begin{aligned}
\left(\lambda_{0} I+A^{\mathrm{hom}}\right)_{h j}=\int_{Y} \lambda \delta_{h j} \mathrm{~d} y+\int_{\Gamma} \beta \delta_{h j} \mathrm{~d} \sigma-\int_{\Gamma} \beta v_{h} v_{j} \mathrm{~d} \sigma & \\
& -\int_{Y} \lambda \nabla \chi_{h} \cdot \nabla \chi_{j} \mathrm{~d} y-\int_{\Gamma} \beta \nabla^{B} \chi_{h} \cdot \nabla^{B} \chi_{j} \mathrm{~d} \sigma \\
= & \int_{Y} \lambda \nabla\left(\chi_{h}-y_{h}\right) \cdot \nabla\left(\chi_{j}-y_{j}\right) \mathrm{d} y+\int_{\Gamma} \beta \nabla^{B}\left(\chi_{h}-y_{h}\right) \cdot \nabla^{B}\left(\chi_{j}-y_{j}\right) \mathrm{d} \sigma .
\end{aligned}
$$


Finally, setting $\lambda_{\min }=\min \left(\lambda_{\text {int }}, \lambda_{\text {out }}\right)$ and using Jensen's inequality, we obtain

$$
\begin{aligned}
& \sum_{h, j=1}^{N}\left(\lambda_{0} I+A^{\mathrm{hom}}\right)_{h j} \xi_{h} \xi_{j}=\int_{Y} \sum_{h, j=1}^{N} \lambda\left(\nabla \chi_{h} \xi_{h}-\boldsymbol{e}_{h} \xi_{h}\right) \cdot\left(\nabla \chi_{j} \xi_{j}-\boldsymbol{e}_{j} \xi_{j}\right) \mathrm{d} y \\
& +\int_{\Gamma} \sum_{h, j=1}^{N} \beta \nabla^{B}\left(\chi_{h} \xi_{h}-y_{h} \xi_{h}\right) \cdot \nabla^{B}\left(\chi_{j} \xi_{j}-y_{j} \xi_{j}\right) \mathrm{d} \sigma \\
& \geq \lambda_{\min } \int_{Y}\left|\sum_{h=1}^{N}\left(\nabla \chi_{h} \xi_{h}-\boldsymbol{e}_{h} \xi_{h}\right)\right|^{2} \mathrm{~d} y+\beta \int_{\Gamma}\left|\sum_{h=1}^{N} \nabla^{B}\left(\chi_{h} \xi_{h}-y_{h} \xi_{h}\right)\right|^{2} \mathrm{~d} \sigma \\
& \geq \lambda_{\min }\left|\int_{Y} \sum_{h=1}^{N}\left(\nabla \chi_{h} \xi_{h}-\boldsymbol{e}_{h} \xi_{h}\right) \mathrm{d} y\right|^{2}+\beta|\Gamma|\left|\frac{1}{|\Gamma|} \int_{\Gamma} \sum_{h=1}^{N} \nabla^{B}\left(\chi_{h} \xi_{h}-y_{h} \xi_{h}\right) \mathrm{d} \sigma\right|^{2} \\
& \geq \lambda_{\min } \sum_{j=1}^{N}\left(\sum_{h=1}^{N}\left(\xi_{h} \int_{Y} \frac{\partial \chi_{h}}{\partial y_{j}} \mathrm{~d} y-\delta_{h j} \xi_{h}\right)\right)^{2}+\frac{\beta}{|\Gamma|}\left|\sum_{h=1}^{N} \int_{\Gamma} \nabla^{B}\left(\chi_{h} \xi_{h}-y_{h} \xi_{h}\right) \mathrm{d} \sigma\right|^{2} \\
& \geq \lambda_{\min } \sum_{j=1}^{N}\left(\sum_{h=1}^{N} \xi_{h} \int_{\partial Y} \chi_{h} n_{j} \mathrm{~d} \sigma-\xi_{j}\right)^{2}=\lambda_{\min }|\xi|^{2}
\end{aligned}
$$

where we have denoted by $n=\left(n_{1}, \ldots, n_{N}\right)$ the outward unit normal to $\partial Y$. Moreover, we remark that the last integral vanishes because of the periodicity of the cell function $\chi_{h}$.

This proves that the homogenized matrix is positive-definite.

Remark 3.2. We note that the homogenized matrix is positive-definite independently of the strict positivity of $\beta$.

Once Theorem 3.1 has been proved, the existence of a unique solution for (3-24) complemented with suitable initial and boundary conditions is standard. The next proposition states the regularity of this solution, which is a property needed in order to obtain the error estimate.

Proposition 3.3. Assume $\bar{u}_{0} \in \mathcal{C}_{c}^{\infty}(\Omega)$ (i.e., $\bar{u}_{0}$ has compact support in $\Omega$ ). Then, the solution $u_{0}$ to (3-24) satisfying the homogeneous boundary condition on $\partial \Omega \times$ $[0, T]$ and the initial condition $u(x, 0)=\bar{u}_{0}(x)$ in $\Omega$ belongs to $\mathcal{C}^{\infty}(\bar{\Omega} \times[0, T])$.

Proof. The result can be obtained applying [Friedman 1964, Theorem 12 in §5].

Remark 3.4. Actually, the asserted $\mathcal{C}^{\infty}$-regularity of the homogenized solution $u_{0}$ is far from being optimal in order to obtain the error estimate proved in Section 4. Indeed, to this purpose, it is enough to have that $u_{0} \in \mathcal{C}^{0}\left([0, T] ; \mathcal{C}^{3}(\bar{\Omega})\right)$ and this is 
guaranteed if, for instance, $\bar{u}_{0} \in \mathcal{C}^{4}(\bar{\Omega})$ and satisfies the compatibility conditions

$$
L_{\mathrm{hom}} \bar{u}_{0}(x)=0, \quad L_{\mathrm{hom}}^{2} \bar{u}_{0}(x):=L_{\mathrm{hom}}\left(L_{\mathrm{hom}} \bar{u}_{0}(x)\right)=0 \quad \text { on } \partial \Omega,
$$

where $L_{\text {hom }}=-\operatorname{div}\left(\left(\lambda_{0} I+A^{\text {hom }}\right) \nabla\right)$, with $\lambda_{0}$ and $A^{\text {hom }}$ defined in (3-25). However, we prefer the simpler assumptions of Proposition 3.3, since we are not interested in stating which are the minimal conditions to be satisfied by the initial data in order to obtain the optimal regularity of the homogenized solution.

For further use (taking into account the system satisfied by $u_{2}$ and (3-24)), we introduce the factorization of the function $u_{2}$ in terms of the homogenized solution $u_{0}$; i.e.,

$$
u_{2}(x, y, t)=\tilde{\chi}_{i j}(y) \frac{\partial^{2} u_{0}}{\partial x_{i} \partial x_{j}}(x, t), \quad i, j=1, \ldots, N,
$$

where the functions $\tilde{\chi}_{i j}: Y \rightarrow \mathbb{R}$ satisfy

$$
\begin{aligned}
&-\lambda \Delta_{y} \tilde{\chi}_{i j}=-\frac{\mu}{\tilde{\mu}}\left(\lambda_{0} \delta_{i j}+a_{i j}^{\mathrm{hom}}\right)+\lambda \delta_{i j}-2 \lambda \frac{\partial \chi_{i}}{\partial y_{j}}=: F \text { in } E_{\text {int }}, E_{\text {out }}, \\
& \beta \Delta_{y}^{B} \tilde{\chi}_{i j}+\left[\lambda \nabla_{y} \tilde{\chi}_{i j} \cdot v\right]=\frac{\alpha}{\tilde{\mu}}\left(\lambda_{0} \delta_{i j}+a_{i j}^{\mathrm{hom}}\right)-\beta\left(\delta_{i j}-(v \otimes v)_{i j}\right) \\
&+2 \beta(I-(v \otimes v))_{i} \cdot \nabla \chi_{j}-\beta v_{j} \chi_{i} \operatorname{div} v+\left[\lambda v_{i}\right] \chi_{j}=: G \text { on } \Gamma, \\
& {\left[\tilde{\chi}_{i j}\right]=0 } \text { on } \Gamma .
\end{aligned}
$$

The functions $\tilde{\chi}_{i j}$ are also required to be periodic in $Y$, with zero integral average on $Y$. In order to obtain (3-30)-(3-32) we have taken into account (3-12), which gives

$$
\operatorname{div}_{x}^{B}\left(\nabla_{y}^{B} \phi\right)+\operatorname{div}_{y}^{B}\left(\nabla_{x}^{B} \phi\right)=2\left(\delta_{i j}-v_{i} v_{j}\right) \frac{\partial^{2} \phi}{\partial x_{i} \partial y_{j}}-v_{j} \frac{\partial v_{i}}{\partial y_{i}} \frac{\partial \phi}{\partial x_{j}},
$$

with $\phi(x, y, t)=u_{1}(x, y, t)=-\chi(y) \cdot \nabla_{x} u_{0}(x, t)$ and the usual summation convention for repeated indexes. By [Amar and Gianni 2018a, Theorem 5.1 and Remark 5.3], problem (3-30)-(3-32) admits a unique solution $\tilde{\chi}_{i j} \in \mathcal{C}_{\#}^{\infty}(Y)$, for $i, j=1, \ldots, N$, since it is easy to check that

$$
\int_{Y} F \mathrm{~d} y=\int_{\Gamma} G \mathrm{~d} \sigma
$$

\section{Error estimate}

In this section we prove that the limit $u$ of the sequence $\left\{u_{\varepsilon}\right\}$ of the solutions of problem (2-6)-(2-10) coincides with the solution $u_{0}$ of (3-24). In order to achieve this result, we will state an error estimate for the sequence $\left\{u_{\varepsilon}\right\}$, which gives the rate of convergence of such a sequence to the homogenized function $u_{0}$, in a suitable norm, thus obtaining a stronger convergence result with respect to the one 
obtained in our previous paper [Amar and Gianni 2018b]. However, this result needs extra-regularity assumptions on the initial data $\bar{u}_{0}(x)$ (see Proposition 3.3 and Remark 3.4), which assure more regularity of the homogenized solution $u_{0}$.

Theorem 4.1. Assume that $\bar{u}_{0} \in \mathcal{C}_{c}^{\infty}(\Omega)$. Let $u_{0}$ be the smooth solution of (3-24), satisfying the initial condition $u_{0}(x, 0)=\bar{u}_{0}(x)$ in $\Omega$ and the boundary condition $u_{0}(x, t)=0$ on $\partial \Omega \times(0, T)$; moreover, let $u_{1}$ be the function defined in (3-13). Then

$$
\begin{aligned}
\left\|u_{\varepsilon}-\left(u_{0}+\varepsilon u_{1}\right)\right\|_{L^{2}\left(0, T ; H^{1}(\Omega)\right)} & \leq \gamma \sqrt{\varepsilon}, \\
\left\|u_{\varepsilon}-u_{0}\right\|_{L^{2}\left(\Omega_{T}\right)} & \leq \gamma \sqrt{\varepsilon},
\end{aligned}
$$

for a proper constant $\gamma>0$, independent of $\varepsilon$.

Proof. Let us define the rest function

$$
r_{\varepsilon}(x, t)=\left(u_{\varepsilon}(x, t)-u_{0}(x, t)-\varepsilon u_{1}(x, x / \varepsilon, t)\right) \varepsilon^{-1}, \quad x \in \Omega, t>0 .
$$

Separately in $\Omega_{\text {int }}^{\varepsilon}$ and in $\Omega_{\text {out }}^{\varepsilon}$, we get

$$
\begin{aligned}
\mu^{\varepsilon} \frac{\partial r_{\varepsilon}}{\partial t}- & \operatorname{div}\left(\lambda^{\varepsilon} \nabla r_{\varepsilon}\right)=\frac{1}{\varepsilon}\left\{-\mu^{\varepsilon} \frac{\partial u_{0}}{\partial t}+\operatorname{div}\left(\lambda^{\varepsilon} \nabla u_{0}\right)-\mu^{\varepsilon} \varepsilon \frac{\partial u_{1}}{\partial t}+\varepsilon \operatorname{div}\left(\lambda^{\varepsilon} \nabla u_{1}\right)\right\} \\
= & \frac{1}{\varepsilon}\left\{-\mu^{\varepsilon} \frac{\partial u_{0}}{\partial t}+\lambda^{\varepsilon} \Delta_{x} u_{0}+2 \lambda^{\varepsilon} u_{\left.1 x_{h} y_{h}\right\}}\right\}-\mu^{\varepsilon} \frac{\partial u_{1}}{\partial t}+\lambda^{\varepsilon} \Delta_{x} u_{1}+\frac{1}{\varepsilon^{2}} \lambda^{\varepsilon} \Delta_{y} u_{1} \\
= & -\frac{1}{\varepsilon} \lambda^{\varepsilon} \Delta_{y} u_{2}-\mu^{\varepsilon} \frac{\partial u_{1}}{\partial t}+\lambda^{\varepsilon} \Delta_{x} u_{1}=: E_{\varepsilon}-\mu^{\varepsilon} \frac{\partial u_{1}}{\partial t} .
\end{aligned}
$$

Moreover,

$\left[r_{\varepsilon}\right]=0, \quad r_{\varepsilon}(x, 0)=-u_{1}(x, x / \varepsilon, 0)=\chi(x / \varepsilon) \cdot \nabla_{x} u_{0}(x, 0)=\chi(x / \varepsilon) \cdot \nabla_{x} \bar{u}_{0}(x, 0)$, and

$$
\begin{aligned}
& \varepsilon \alpha \frac{\partial r_{\varepsilon}}{\partial t}-\varepsilon \beta \Delta^{B} r_{\varepsilon}=\frac{1}{\varepsilon}\left\{\varepsilon \alpha \frac{\partial u_{\varepsilon}}{\partial t}-\varepsilon \beta \Delta^{B} u_{\varepsilon}-\varepsilon \alpha \frac{\partial u_{0}}{\partial t}+\varepsilon \beta \Delta^{B} u_{0}\right\}-\left\{\varepsilon \alpha \frac{\partial u_{1}}{\partial t}-\varepsilon \beta \Delta^{B} u_{1}\right\} \\
& =\frac{1}{\varepsilon}\left[\lambda^{\varepsilon} \nabla u_{\varepsilon} \cdot v_{\varepsilon}\right]-\alpha \frac{\partial u_{0}}{\partial t}+\beta \Delta_{x}^{B} u_{0}+\beta \operatorname{div}_{x}^{B} \nabla_{y}^{B} u_{1}+\beta \operatorname{div}_{y}^{B} \nabla_{x}^{B} u_{1} \\
& -\varepsilon \alpha \frac{\partial u_{1}}{\partial t}+\varepsilon \beta \Delta_{x}^{B} u_{1}+\frac{1}{\varepsilon}\left(\beta \Delta_{y}^{B} u_{1}+\beta \operatorname{div}_{y}^{B} \nabla_{x}^{B} u_{0}+\beta \operatorname{div}_{x}^{B} \nabla_{y}^{B} u_{0}\right)+\frac{1}{\varepsilon^{2}} \beta \Delta_{y}^{B} u_{0} \\
& =\frac{1}{\varepsilon}\left[\lambda^{\varepsilon} \nabla u_{\varepsilon} \cdot v_{\varepsilon}\right]-\left[\lambda^{\varepsilon}\left(\nabla_{x} u_{1}+\nabla_{y} u_{2}\right) \cdot v_{\varepsilon}\right]-\beta \Delta_{y}^{B} u_{2} \\
& -\varepsilon\left(\alpha \frac{\partial u_{1}}{\partial t}-\beta \Delta_{x}^{B} u_{1}\right)-\frac{1}{\varepsilon}\left[\lambda^{\varepsilon}\left(\nabla_{x} u_{0}+\nabla_{y} u_{1}\right) \cdot v_{\varepsilon}\right] \\
& =\left[\lambda^{\varepsilon} \nabla r_{\varepsilon} \cdot v_{\varepsilon}\right]-\varepsilon\left(\alpha \frac{\partial u_{1}}{\partial t}-\beta \Delta_{x}^{B} u_{1}\right)-\left[\lambda^{\varepsilon} \nabla_{y} u_{2} \cdot v_{\varepsilon}\right]-\beta \Delta_{y}^{B} u_{2},
\end{aligned}
$$


where we have taken into account the problems satisfied by $u_{1}$ and $u_{2}\left(u_{1}\right.$ and $u_{2}$ are defined in Section 3.1) and the fact that $\operatorname{div}_{x}^{B} \nabla_{y}^{B} u_{0}=0$ and $\Delta_{y}^{B} u_{0}=0$.

Let us now introduce the corrected rest function

$$
\tilde{r}_{\varepsilon}=r_{\varepsilon}+u_{1} \phi_{\varepsilon}
$$

where $\phi_{\varepsilon}$ is a cut-off function equal to 1 in a neighborhood of $\partial \Omega$, and such that

$$
\phi_{\varepsilon}(x)=0 \quad \text { if } \operatorname{dist}(x, \partial \Omega) \geq \gamma_{0} \varepsilon .
$$

Clearly, $\phi_{\varepsilon} \equiv 0$ on $\Gamma^{\varepsilon}$ (since $\operatorname{dist}\left(\Gamma^{\varepsilon}, \partial \Omega\right) \geq \gamma_{0} \varepsilon$, by the assumptions made in Section 2.2), so that $r_{\varepsilon}=\tilde{r}_{\varepsilon}$ on $\Gamma^{\varepsilon}$. We may assume $0 \leq \phi_{\varepsilon} \leq 1,\left|\nabla \phi_{\varepsilon}\right| \leq \gamma / \varepsilon$. The function $\tilde{r}_{\varepsilon}$ satisfies $\left[\tilde{r}_{\varepsilon}\right]=0$ on $\Gamma^{\varepsilon}$ and

$$
\begin{aligned}
\mu^{\varepsilon} \frac{\partial \tilde{r}_{\varepsilon}}{\partial t}-\lambda^{\varepsilon} \Delta \tilde{r}_{\varepsilon} & =E_{\varepsilon}-\mu^{\varepsilon} \frac{\partial u_{1}}{\partial t}+\mu^{\varepsilon} \phi_{\varepsilon} \frac{\partial u_{1}}{\partial t}-\lambda^{\varepsilon} \Delta\left(u_{1} \phi_{\varepsilon}\right) & & \text { in } \Omega_{\text {int }}^{\varepsilon}, \Omega_{\text {out }}^{\varepsilon}, \\
\tilde{r}_{\varepsilon}(x, 0) & =\chi(x / \varepsilon) \cdot \nabla_{x} \bar{u}_{0}(x, 0)\left(1-\phi_{\varepsilon}\right) & & \text { on } \Omega, \\
\tilde{r}_{\varepsilon} & =0 & & \text { on } \partial \Omega,
\end{aligned}
$$

and on $\Gamma^{\varepsilon}$

$$
\begin{gathered}
\varepsilon \alpha \frac{\partial \tilde{r}_{\varepsilon}}{\partial t}-\varepsilon \beta \Delta^{B} \tilde{r}_{\varepsilon}=\left[\lambda^{\varepsilon} \nabla r_{\varepsilon} \cdot v_{\varepsilon}\right]-\varepsilon\left(\alpha \frac{\partial u_{1}}{\partial t}-\beta \Delta_{x}^{B} u_{1}\right)-\left[\lambda^{\varepsilon} \nabla_{y} u_{2} \cdot v_{\varepsilon}\right]-\beta \Delta_{y}^{B} u_{2} \\
=\left[\lambda^{\varepsilon} \nabla \tilde{r}_{\varepsilon} \cdot v_{\varepsilon}\right]-\varepsilon\left(\alpha \frac{\partial u_{1}}{\partial t}-\beta \Delta_{x}^{B} u_{1}\right)-\left[\lambda^{\varepsilon} \nabla_{y} u_{2} \cdot v_{\varepsilon}\right]-\beta \Delta_{y}^{B} u_{2}
\end{gathered}
$$

Note that the correction $u_{1} \phi_{\varepsilon}$ has been introduced precisely in order to guarantee (4-5). Multiply (4-3) by $\tilde{r}_{\varepsilon}$ and integrate by parts; by virtue of (4-5), we get

$$
\begin{aligned}
& \int_{0}^{t} \int_{\Omega}\left\{E_{\varepsilon}-\lambda^{\varepsilon} \Delta\left(u_{1} \phi_{\varepsilon}\right)\right\} \tilde{r}_{\varepsilon} \mathrm{d} x \mathrm{~d} \tau-\int_{0}^{t} \int_{\Omega}\left\{\mu^{\varepsilon} \frac{\partial u_{1}}{\partial \tau}\left(1-\phi_{\varepsilon}\right)\right\} \tilde{r}_{\varepsilon} \mathrm{d} x \mathrm{~d} \tau \\
& =\frac{1}{2} \int_{0}^{t} \int_{\Omega} \mu^{\varepsilon} \frac{\partial \tilde{r}_{\varepsilon}^{2}}{\partial \tau} \mathrm{d} x \mathrm{~d} \tau+\int_{0}^{t} \int_{\Omega} \lambda^{\varepsilon}\left|\nabla \tilde{r}_{\varepsilon}\right|^{2} \mathrm{~d} x \mathrm{~d} \tau+\int_{0}^{t} \int_{\Gamma^{\varepsilon}}\left[\lambda^{\varepsilon} \nabla \tilde{r}_{\varepsilon} \cdot v_{\varepsilon}\right] \tilde{r}_{\varepsilon} \mathrm{d} \sigma \mathrm{d} \tau \\
& =\frac{1}{2} \int_{\Omega} \mu^{\varepsilon} \tilde{r}_{\varepsilon}^{2}(x, t) \mathrm{d} x-\frac{1}{2} \int_{\Omega} \mu^{\varepsilon} \tilde{r}_{\varepsilon}^{2}(x, 0) \mathrm{d} x+\int_{0}^{t} \int_{\Omega} \lambda^{\varepsilon}\left|\nabla \tilde{r}_{\varepsilon}\right|^{2} \mathrm{~d} x \mathrm{~d} \tau \\
& +\frac{\varepsilon}{2} \int_{\Gamma^{\varepsilon}} \alpha \tilde{r}_{\varepsilon}^{2}(x, t) \mathrm{d} \sigma-\frac{\varepsilon}{2} \int_{\Gamma^{\varepsilon}} \alpha \tilde{r}_{\varepsilon}^{2}(x, 0) \mathrm{d} \sigma+\varepsilon \beta \int_{0}^{t} \int_{\Gamma^{\varepsilon}}\left|\nabla^{B} \tilde{r}_{\varepsilon}\right|^{2} \mathrm{~d} \sigma \mathrm{d} \tau \\
& +\varepsilon \int_{0}^{t} \int_{\Gamma^{\varepsilon}}\left(\alpha \frac{\partial u_{1}}{\partial t}-\beta \Delta_{x}^{B} u_{1}\right) \tilde{r}_{\varepsilon} \mathrm{d} \sigma \mathrm{d} \tau+\int_{0}^{t} \int_{\Gamma^{\varepsilon}}\left(\beta \Delta_{y}^{B} u_{2}+\left[\lambda \nabla_{y} u_{2} \cdot v_{\varepsilon}\right]\right) \tilde{r}_{\varepsilon} \mathrm{d} \sigma \mathrm{d} \tau .
\end{aligned}
$$


This implies

$$
\begin{aligned}
\frac{1}{2} \int_{\Omega} \mu^{\varepsilon} \tilde{r}_{\varepsilon}^{2}(x, t) \mathrm{d} x+\frac{\varepsilon}{2} \int_{\Gamma^{\varepsilon}} \alpha \tilde{r}_{\varepsilon}^{2}(x, t) \mathrm{d} \sigma+\int_{0}^{t} \int_{\Omega} \lambda^{\varepsilon}\left|\nabla \tilde{r}_{\varepsilon}\right|^{2} \mathrm{~d} x \mathrm{~d} \tau \\
\quad+\varepsilon \beta \int_{0}^{t} \int_{\Gamma^{\varepsilon}}\left|\nabla^{B} \tilde{r}_{\varepsilon}\right|^{2} \mathrm{~d} \sigma \mathrm{d} \tau \\
=\frac{1}{2} \int_{\Omega} \mu^{\varepsilon} \tilde{r}_{\varepsilon}^{2}(x, 0) \mathrm{d} x+\frac{\varepsilon}{2} \int_{\Gamma^{\varepsilon}} \alpha \tilde{r}_{\varepsilon}^{2}(x, 0) \mathrm{d} \sigma \\
\quad-\varepsilon \int_{0}^{t} \int_{\Gamma^{\varepsilon}}\left(\alpha \frac{\partial u_{1}}{\partial \tau}-\beta \Delta_{x}^{B} u_{1}\right) \tilde{r}_{\varepsilon} \mathrm{d} \sigma \mathrm{d} \tau \\
\quad-\int_{0}^{t} \int_{\Gamma^{\varepsilon}}\left(\beta \Delta_{y}^{B} u_{2}+\left[\lambda^{\varepsilon} \nabla_{y} u_{2} \cdot v_{\varepsilon}\right]\right) \tilde{r}_{\varepsilon} \mathrm{d} \sigma \mathrm{d} \tau \\
\quad+\int_{0}^{t} \int_{\Omega}\left\{E_{\varepsilon}-\lambda^{\varepsilon} \Delta\left(u_{1} \phi_{\varepsilon}\right)\right\} \tilde{r}_{\varepsilon} \mathrm{d} x \mathrm{~d} \tau-\int_{0}^{t} \int_{\Omega}\left\{\mu^{\varepsilon} \frac{\partial u_{1}}{\partial \tau}\left(1-\phi_{\varepsilon}\right)\right\} \tilde{r}_{\varepsilon} \mathrm{d} x \mathrm{~d} \tau .
\end{aligned}
$$

Next, compute

$$
\begin{aligned}
& \int_{0}^{t} \int_{\Omega} E_{\varepsilon} \tilde{r}_{\varepsilon} \mathrm{d} x \mathrm{~d} \tau=\int_{0}^{t} \int_{\Omega} \lambda^{\varepsilon}\left\{-\frac{1}{\varepsilon} \Delta_{y} u_{2}+\Delta_{x} u_{1}\right\} \tilde{r}_{\varepsilon} \mathrm{d} x \mathrm{~d} \tau \\
& =\int_{0}^{t} \int_{\Omega} \lambda^{\varepsilon}\left\{-\frac{1}{\varepsilon} \Delta_{y} u_{2}-\operatorname{div}_{x}\left(\nabla_{y} u_{2}\right)\right\} \tilde{r}_{\varepsilon} \mathrm{d} x \mathrm{~d} \tau \\
& \quad+\int_{0}^{t} \int_{\Omega} \lambda^{\varepsilon}\left\{\operatorname{div}_{x}\left(\nabla_{y} u_{2}\right)+\Delta_{x} u_{1}\right\} \tilde{r}_{\varepsilon} \mathrm{d} x \mathrm{~d} \tau \\
& =-\int_{0}^{t} \int_{\Omega} \operatorname{div}\left(\lambda^{\varepsilon} \nabla_{y} u_{2}\right) \tilde{r}_{\varepsilon} \mathrm{d} x \mathrm{~d} \tau \\
& \quad+\int_{0}^{t} \int_{\Omega}\left\{\lambda^{\varepsilon} \operatorname{div}_{x}\left(\nabla_{y} u_{2}\right)+\lambda^{\varepsilon} \Delta_{x} u_{1}\right\} \tilde{r}_{\varepsilon} \mathrm{d} x \mathrm{~d} \tau \\
& =\int_{0}^{t} \int_{\Gamma^{\varepsilon}}\left[\lambda^{\varepsilon} \nabla_{y} u_{2} \cdot v_{\varepsilon}\right] \tilde{r}_{\varepsilon} \mathrm{d} \sigma \mathrm{d} \tau+\int_{0}^{t} \int_{\Omega} \lambda^{\varepsilon} \nabla_{y} u_{2} \cdot \nabla \tilde{r}_{\varepsilon} \mathrm{d} x \mathrm{~d} \tau \\
& \quad+\int_{0}^{t} \int_{\Omega}\left\{\lambda^{\varepsilon} \operatorname{div}_{x}\left(\nabla_{y} u_{2}\right)+\lambda^{\varepsilon} \Delta_{x} u_{1}\right\} \tilde{r}_{\varepsilon} \mathrm{d} x \mathrm{~d} \tau .
\end{aligned}
$$

Note that the last integral in (4-8) can be bounded in the following way:

$$
\int_{0}^{t} \int_{\Omega}\left\{\lambda^{\varepsilon} \operatorname{div}_{x}\left(\nabla_{y} u_{2}\right)+\lambda^{\varepsilon} \Delta_{x} u_{1}\right\} \tilde{r}_{\varepsilon} \mathrm{d} x \mathrm{~d} \tau \leq \gamma(\delta)+\delta \int_{0}^{t} \int_{\Omega} \tilde{r}_{\varepsilon}^{2} \mathrm{~d} x \mathrm{~d} \tau,
$$


where $\delta>0$ will be chosen in the following. We exploit here the estimate

$$
\int_{0}^{t} \int_{\Omega}\left(u_{2 x_{i} y_{i}}^{2}+u_{1 x_{i} x_{i}}^{2}\right) \mathrm{d} x \mathrm{~d} \tau \leq \gamma
$$

which is a consequence of the regularity of the cell functions $\chi$ and $\tilde{\chi}$ (recall (3-13)-(3-16) and (3-29)-(3-32)) and of the homogenized function $u_{0}$. Similarly, for $\delta^{\prime}=\min \left(\lambda_{\text {int }}, \lambda_{\text {out }}\right) / 2$,

$$
\begin{gathered}
-\int_{0}^{t} \int_{\Omega} \lambda^{\varepsilon} \Delta\left(u_{1} \phi_{\varepsilon}\right) \tilde{r}_{\varepsilon} \mathrm{d} x \mathrm{~d} \tau=\int_{0}^{t} \int_{\Omega} \lambda^{\varepsilon} \nabla\left(u_{1} \phi_{\varepsilon}\right) \cdot \nabla \tilde{r}_{\varepsilon} \mathrm{d} x \mathrm{~d} \tau \\
\leq \delta^{\prime} \int_{0}^{t} \int_{\Omega}\left|\nabla \tilde{r}_{\varepsilon}\right|^{2} \mathrm{~d} x \mathrm{~d} \tau+\frac{\gamma\left(\delta^{\prime}\right)}{\varepsilon^{2}}\left|\left\{x \in \Omega \mid \operatorname{dist}(x, \partial \Omega) \leq \gamma_{0} \varepsilon\right\}\right| \\
\leq \delta^{\prime} \int_{0}^{t} \int_{\Omega}\left|\nabla \tilde{r}_{\varepsilon}\right|^{2} \mathrm{~d} x \mathrm{~d} \tau+\frac{\gamma\left(\delta^{\prime}\right)}{\varepsilon}
\end{gathered}
$$

where, again due to the stated regularity of $\chi$ and $u_{0}$, we used

$$
\sup _{x \in \Omega, y \in Y, 0<t<T}\left\{\left|u_{1}\right|+\left|\nabla_{x} u_{1}\right|+\left|\nabla_{y} u_{1}\right|\right\}(x, y, t)<+\infty .
$$

Moreover, for $\delta^{\prime \prime}$ which will be chosen later, we obtain

$$
\begin{array}{r}
\int_{0}^{t} \int_{\Gamma^{\varepsilon}}\left(\beta \Delta_{y}^{B} u_{2}\right) \tilde{r}_{\varepsilon} \mathrm{d} \sigma \mathrm{d} \tau=\varepsilon \beta \int_{0}^{t} \int_{\Gamma^{\varepsilon}}\left(\frac{1}{\varepsilon} \operatorname{div}_{y}^{B} \nabla_{y}^{B} u_{2}+\operatorname{div}_{x}^{B} \nabla_{y}^{B} u_{2}\right) \tilde{r}_{\varepsilon} \mathrm{d} \sigma \mathrm{d} \tau \\
-\varepsilon \beta \int_{0}^{t} \int_{\Gamma^{\varepsilon}}\left(\operatorname{div}_{x}^{B} \nabla_{y}^{B} u_{2}\right) \tilde{r}_{\varepsilon} \mathrm{d} \sigma \mathrm{d} \tau \\
=-\varepsilon \beta \int_{0}^{t} \int_{\Gamma^{\varepsilon}} \nabla_{y}^{B} u_{2} \nabla^{B} \tilde{r}_{\varepsilon} \mathrm{d} \sigma \mathrm{d} \tau-\varepsilon \beta \int_{0}^{t} \int_{\Gamma^{\varepsilon}}\left(\operatorname{div}_{x}^{B} \nabla_{y}^{B} u_{2}\right) \tilde{r}_{\varepsilon} \mathrm{d} \sigma \mathrm{d} \tau \\
=\gamma\left(\delta^{\prime \prime}\right)+\delta^{\prime \prime} \varepsilon \int_{0}^{t} \int_{\Gamma^{\varepsilon}}\left|\nabla^{B} \tilde{r}_{\varepsilon}\right|^{2} \mathrm{~d} \sigma \mathrm{d} \tau+\gamma\left(\delta^{\prime \prime}\right)+\delta^{\prime \prime} \varepsilon \int_{0}^{t} \int_{\Gamma^{\varepsilon}} \tilde{r}_{\varepsilon}^{2} \mathrm{~d} \sigma \mathrm{d} \tau .
\end{array}
$$

Here, we use

$$
\varepsilon \int_{0}^{t} \int_{\Gamma^{\varepsilon}}\left(\left|\nabla_{y}^{B} u_{2}\right|^{2}+\left|\operatorname{div}_{x}^{B} \nabla_{y}^{B} u_{2}\right|^{2}\right) \mathrm{d} \sigma \mathrm{d} \tau \leq \gamma
$$

which is again a consequence of the regularity of $\tilde{\chi}$ and $u_{0}$. 
Combining the previous estimates, we have

$$
\begin{aligned}
\frac{1}{2} \int_{\Omega} \mu^{\varepsilon} \tilde{r}_{\varepsilon}^{2}(x, t) \mathrm{d} x+\frac{\varepsilon}{2} \int_{\Gamma^{\varepsilon}} \alpha \tilde{r}_{\varepsilon}^{2}(x, t) \mathrm{d} \sigma+\int_{0}^{t} \int_{\Omega} \lambda^{\varepsilon}\left|\nabla \tilde{r}_{\varepsilon}\right|^{2} \mathrm{~d} x \mathrm{~d} \tau \\
\quad+\varepsilon \beta \int_{0}^{t} \int_{\Gamma^{\varepsilon}}\left|\nabla^{B} \tilde{r}_{\varepsilon}\right|^{2} \mathrm{~d} \sigma \mathrm{d} \tau \\
\leq \frac{1}{2} \int_{\Omega} \mu^{\varepsilon} \tilde{r}_{\varepsilon}^{2}(x, 0) \mathrm{d} x+\frac{\varepsilon}{2} \int_{\Gamma^{\varepsilon}} \alpha \tilde{r}_{\varepsilon}^{2}(x, 0) \mathrm{d} \sigma-\varepsilon \int_{0}^{t} \int_{\Gamma^{\varepsilon}}\left(\alpha \frac{\partial u_{1}}{\partial \tau}-\beta \Delta_{x}^{B} u_{1}\right) \tilde{r}_{\varepsilon} \mathrm{d} \sigma \mathrm{d} \tau \\
+\gamma\left(\delta^{\prime \prime}\right)+\delta^{\prime \prime} \varepsilon \int_{0}^{t} \int_{\Gamma^{\varepsilon}}\left|\nabla^{B} \tilde{r}_{\varepsilon}\right|^{2} \mathrm{~d} \sigma \mathrm{d} \tau+\delta^{\prime \prime} \varepsilon \int_{0}^{t} \int_{\Gamma^{\varepsilon}} \tilde{r}_{\varepsilon}^{2} \mathrm{~d} \sigma \mathrm{d} \tau \\
\quad-\int_{0}^{t} \int_{\Gamma^{\varepsilon}}\left[\lambda^{\varepsilon} \nabla_{y} u_{2} \cdot v_{\varepsilon}\right] \tilde{r}_{\varepsilon} \mathrm{d} \sigma \mathrm{d} \tau+\int_{0}^{t} \int_{\Gamma^{\varepsilon}}\left[\lambda^{\varepsilon} \nabla_{y} u_{2} \cdot v_{\varepsilon}\right] \tilde{r}_{\varepsilon} \mathrm{d} \sigma \mathrm{d} \tau \\
+\int_{0}^{t} \int_{\Omega} \lambda^{\varepsilon} \nabla_{y} u_{2} \cdot \nabla \tilde{r}_{\varepsilon} \mathrm{d} x \mathrm{~d} \tau+\gamma(\delta)+\delta \int_{0}^{t} \int_{\Omega} \tilde{r}_{\varepsilon}^{2} \mathrm{~d} x \mathrm{~d} \tau \\
+\delta^{\prime} \int_{0}^{t} \int_{\Omega}\left|\nabla \tilde{r}_{\varepsilon}\right|^{2} \mathrm{~d} x \mathrm{~d} \tau+\frac{\gamma\left(\delta^{\prime}\right)}{\varepsilon}-\int_{0}^{t} \int_{\Omega}\left\{\mu^{\varepsilon} \frac{\partial u_{1}}{\partial \tau}\left(1-\phi_{\varepsilon}\right)\right\} \tilde{r}_{\varepsilon} \mathrm{d} x \mathrm{~d} \tau \\
\leq \gamma+\gamma\left(\delta^{\prime \prime \prime}\right)+\varepsilon \delta^{\prime \prime \prime} \int_{0}^{t} \int_{\Gamma^{\varepsilon}} \tilde{r}_{\varepsilon}^{2} \mathrm{~d} \sigma \mathrm{d} \tau \\
+\gamma\left(\delta^{\prime \prime}\right)+\delta^{\prime \prime} \varepsilon \int_{0}^{t} \int_{\Gamma^{\varepsilon}}\left|\nabla^{B} \tilde{r}_{\varepsilon}\right|^{2} \mathrm{~d} \sigma \mathrm{d} \tau+\delta^{\prime \prime} \varepsilon \int_{0}^{t} \int_{\Gamma^{\varepsilon}} \tilde{r}_{\varepsilon}^{2} \mathrm{~d} \sigma \mathrm{d} \tau \\
+\gamma\left(\delta^{\prime \prime \prime}\right)+\delta^{\prime \prime \prime} \int_{0}^{t} \int_{\Omega}\left|\nabla \tilde{r}_{\varepsilon}\right|^{2} \mathrm{~d} x \mathrm{~d} \tau+\gamma(\delta)+\delta \int_{0}^{t} \int_{\Omega} \tilde{r}_{\varepsilon}^{2} \mathrm{~d} x \mathrm{~d} \tau \\
+\delta^{\prime} \int_{0}^{t} \int_{\Omega}\left|\nabla \tilde{r}_{\varepsilon}\right|^{2} \mathrm{~d} x \mathrm{~d} \tau+\frac{\gamma\left(\delta^{\prime}\right)}{\varepsilon}+\gamma\left(\delta^{\prime \prime \prime}\right)+\delta^{\prime \prime \prime} \int_{0}^{t} \int_{\Omega} \tilde{r}_{\varepsilon}^{2} \mathrm{~d} x \mathrm{~d} \tau, \quad(4-12)
\end{aligned}
$$

where $\delta^{\prime \prime \prime}$ will be chosen later. Finally, using Poincaré's inequality, Gronwall's lemma, and absorbing the gradient term in (4-12) into the left-hand side (which is possible choosing $\delta, \delta^{\prime}, \delta^{\prime \prime}, \delta^{\prime \prime \prime}$ sufficiently small), we get

$$
\int_{0}^{t} \int_{\Omega}\left|\nabla \tilde{r}_{\varepsilon}\right|^{2} \mathrm{~d} x \mathrm{~d} \tau \leq \frac{\gamma}{\varepsilon}
$$

On recalling the definition of $\tilde{r}_{\varepsilon}$, and invoking Poincaré's inequality again, we obtain

$$
\int_{0}^{t} \int_{\Omega}\left(u_{\varepsilon}-u_{0}-\varepsilon u_{1}\left(1-\phi_{\varepsilon}\right)\right)^{2} \mathrm{~d} x \mathrm{~d} \tau \leq \gamma \varepsilon
$$

Moreover, taking into account that $r_{\varepsilon}=\tilde{r}_{\varepsilon}-u_{1} \phi_{\varepsilon}$ and using (4-13), it follows that

$$
\int_{0}^{t} \int_{\Omega}\left|\nabla r_{\varepsilon}\right|^{2} \mathrm{~d} x \mathrm{~d} \tau \leq \gamma\left[\int_{0}^{t} \int_{\Omega}\left|\nabla \tilde{r}_{\varepsilon}\right|^{2} \mathrm{~d} x \mathrm{~d} \tau+\int_{0}^{t} \int_{\Omega}\left|\nabla\left(u_{1} \phi_{\varepsilon}\right)\right|^{2} \mathrm{~d} x \mathrm{~d} \tau\right] \leq \frac{\gamma}{\varepsilon},
$$


where we recall the estimate for $\nabla\left(u_{1} \phi_{\varepsilon}\right)$ done in (4-10). Hence, by (4-14) and (4-15), we obtain (4-1). Finally, (4-2) can be obtained making use of (4-14) and taking into account that

$$
\int_{0}^{t} \int_{\Omega}\left(\varepsilon u_{1}\left(1-\phi_{\varepsilon}\right)\right)^{2} \mathrm{~d} x \mathrm{~d} \tau \leq \gamma \varepsilon^{2}
$$

\section{References}

[Allaire and Murat 1993] G. Allaire and F. Murat, "Homogenization of the Neumann problem with nonisolated holes", Asymptotic Anal. 7:2 (1993), 81-95.

[Allaire et al. 1996] G. Allaire, A. Damlamian, and U. Hornung, "Two-scale convergence on periodic surfaces and applications", pp. 15-25 in Mathematical modelling of flow through porous media: proceedings of the conference (Saint-Étienne, France, 1995), edited by A. P. Bourgeat et al., World Scientific, 1996.

[Amar and Gianni 2018a] M. Amar and R. Gianni, "Existence, uniqueness and concentration for a system of PDEs involving the Laplace-Beltrami operator", preprint, 2018. Submitted.

[Amar and Gianni 2018b] M. Amar and R. Gianni, "Laplace-Beltrami operator for the heat conduction in polymer coating of electronic devices", preprint, 2018. To appear in Discrete Cont. Dyn. Sys. $B$.

[Amar et al. 2003] M. Amar, D. Andreucci, P. Bisegna, and R. Gianni, "Evolution and memory effects in the homogenization limit for electrical conduction in biological tissues: the 1-d case", in Proceedings of the 16th AIMETA Congress of Theoretical and Applied Mechanics (Ferrara, Italy, 2003), 2003.

[Amar et al. 2004] M. Amar, D. Andreucci, P. Bisegna, and R. Gianni, "Evolution and memory effects in the homogenization limit for electrical conduction in biological tissues", Math. Models Methods Appl. Sci. 14:9 (2004), 1261-1295.

[Amar et al. 2006] M. Amar, D. Andreucci, P. Bisegna, and R. Gianni, "On a hierarchy of models for electrical conduction in biological tissues”, Math. Methods Appl. Sci. 29:7 (2006), 767-787.

[Amar et al. 2009a] M. Amar, D. Andreucci, P. Bisegna, and R. Gianni, "Exponential asymptotic stability for an elliptic equation with memory arising in electrical conduction in biological tissues", European J. Appl. Math. 20:5 (2009), 431-459.

[Amar et al. 2009b] M. Amar, D. Andreucci, P. Bisegna, and R. Gianni, "Stability and memory effects in a homogenized model governing the electrical conduction in biological tissues", J. Mech. Mater. Struct. 4:2 (2009), 211-223.

[Amar et al. 2010] M. Amar, D. Andreucci, P. Bisegna, and R. Gianni, "Homogenization limit and asymptotic decay for electrical conduction in biological tissues in the high radiofrequency range", Commun. Pure Appl. Anal. 9:5 (2010), 1131-1160.

[Amar et al. 2013] M. Amar, D. Andreucci, P. Bisegna, and R. Gianni, "A hierarchy of models for the electrical conduction in biological tissues via two-scale convergence: the nonlinear case", Differential Integral Equations 26:9-10 (2013), 885-912.

[Amar et al. 2017a] M. Amar, D. Andreucci, and D. Bellaveglia, "The time-periodic unfolding operator and applications to parabolic homogenization", Atti Accad. Naz. Lincei Rend. Lincei Mat. Appl. 28:4 (2017), 663-700.

[Amar et al. 2017b] M. Amar, D. Andreucci, and D. Bellaveglia, "Homogenization of an alternating Robin-Neumann boundary condition via time-periodic unfolding", Nonlinear Anal. Theory Meth. Appl. 153 (2017), 56-77. 
[Andreucci et al. 2003] D. Andreucci, P. Bisegna, and E. DiBenedetto, "Homogenization and concentrated capacity for the heat equation with non-linear variational data in reticular almost disconnected structures and applications to visual transduction", Ann. Mat. Pura Appl. (4) 182:4 (2003), 375-407.

[Auriault and Ene 1994] J.-L. Auriault and H. I. Ene, "Macroscopic modelling of heat transfer in composites with interfacial thermal barrier", Int. J. Heat Mass. Tran. 37:18 (1994), 2885-2892.

[Bensoussan et al. 1978] A. Bensoussan, J.-L. Lions, and G. Papanicolaou, Asymptotic analysis for periodic structures, Studies in Mathematics and its Applications 5, North-Holland, 1978.

[Bunoiu and Timofte 2016] R. Bunoiu and C. Timofte, "Homogenization of a thermal problem with flux jump", Netw. Heterog. Media 11:4 (2016), 545-562.

[Cioranescu et al. 2012] D. Cioranescu, A. Damlamian, P. Donato, G. Griso, and R. Zaki, "The periodic unfolding method in domains with holes", SIAM J. Math. Anal. 44:2 (2012), 718-760.

[Donato and Monsurrò 2001] P. Donato and S. Monsurrò, "Homogenization of two heat conductors with an interfacial contact resistance", Ric. Mat. 50 (2001), 115-144.

[Friedman 1964] A. Friedman, Partial differential equations of parabolic type, Prentice-Hall, 1964.

[Hummel 2000] H.-K. Hummel, "Homogenization for heat transfer in polycrystals with interfacial resistances", Appl. Anal. 75:3-4 (2000), 403-424.

[Jose 2009] E. C. Jose, "Homogenization of a parabolic problem with an imperfect interface", Rev. Roumaine Math. Pures Appl. 54:3 (2009), 189-222.

[Sánchez-Palencia 1980] E. Sánchez-Palencia, Nonhomogeneous media and vibration theory, Lecture Notes in Physics 127, Springer, 1980.

[Timofte 2013] C. Timofte, "Multiscale analysis of diffusion processes in composite media", Comput. Math. Appl. 66:9 (2013), 1573-1580.

Received 25 Sep 2017. Revised 27 Dec 2017. Accepted 17 Feb 2018.

MiCOL AMAR: micol.amar@sbai.uniroma1.it

Dipartimento di Scienze di Base e Applicate per l'Ingegneria, Sapienza Università di Roma, Roma, Italy

ROBERTO GIANNI: roberto.gianni@unifi.it

Dipartimento di Matematica e Informatica, Università di Firenze, Firenze, Italy 